\title{
Fas-Associated Factor 1 as a Regulator of Olfactory Axon Guidance
}

\author{
Kai Cheng, Li Bai, and Leonardo Belluscio \\ Developmental Neural Plasticity Unit, National Institute of Neurological Disorders and Stroke, National Institutes of Health, Bethesda, Maryland 20892
}

Axon guidance is a crucial part of neural circuit formation. While precise axonal targeting forms the basis of accurate information delivery, the mechanisms that regulate this process are still unclear. Apoptotic signaling molecules have been identified in the axon terminal, but their specific role in axon guidance is not well understood. Here we use the mouse olfactory system as an in vivo model to demonstrate that by modulating Fas-associated factor 1 (FAF1), an apoptosis regulatory molecule, we can rewire axonal projections. Interestingly, FAF1 is highly expressed in the developing mouse olfactory system, but its expression is downregulated postnatally. Using a tetracycline-inducible promoter Tet-Off system, we generated transgenic mice in which FAF1 is specifically expressed in immature olfactory sensory neurons (OSNs) and show that overexpression of FAF1 not only misroutes OSN axons to deep layers of the olfactory bulb but also leads to widespread disruption of the glomerular layer. In addition, we also demonstrate that the specific convergence of $\mathrm{P} 2$ receptor OSN axons is completely distorted in the FAF1 mice. Strikingly, all of the mutant phenotypes can be recovered by shutting down FAF1 expression through the administration of doxycycline. Together, our study provides clear in vivo evidence that an apoptotic molecule can indeed regulate axon targeting and that OSNs can restore their organization even after broad disruption.

\section{Introduction}

Axon guidance is a fundamental process in the development of the nervous system. The mammalian olfactory system provides an ideal in vivo model for the study of axon guidance as it forms precise stereotypic projections between the olfactory epithelium $(\mathrm{OE})$ and the olfactory bulb (OB). The accuracy of these projections is based upon the following two basic principles: (1) axons of OSNs target only a specific layer of the OB, the glomerular layer (GL); and (2) all olfactory sensory neurons (OSNs) expressing the same odorant receptor extend their axons to the same subset of glomeruli within that layer, creating a glomerular map that reflects odorant receptor identity on the surface of the $\mathrm{OB}$ (Axel, 1995). Thus, the precision of this map may be used as an in vivo assay to evaluate changes in axon targeting against a predictable pattern. Interestingly, the circuitry that underlies this organization is constantly challenged since the OE continuously regenerates. When mature OSNs are lost, basal cells proliferate, giving rise to new OSNs that accurately target their axons to the OB. This feature provides a unique opportunity to observe axonal projections beyond prenatal development and makes the

\footnotetext{
Received Jan. 4, 2011; revised June 23, 2011; accepted June 29, 2011.

Author contributions: K.C. and L. Belluscio designed research; K.C. and L. Bai performed research; K.C. and L. Belluscio analyzed data; K.C. and L. Belluscio wrote the paper.

This work was supported by the National Institutes of Health, Intramural Research Program, Project 1ZIANS003116-01. We thank the Ryba laboratory for their generosity with transgenic lines, James Pickel at the National Institute of Mental Health Transgenic Facility for pronuclear injection and generation of Tet0-FAF1 mice, and members of the Belluscio laboratory for helpful comments on the manuscript.

Correspondence should be addressed to Dr. Leonardo Belluscio, Developmental Neural Plasticity Unit, National Institute of Neurological Disorders and Stroke, Porter Neuroscience Research Center, Building 35, Room 3A-116, 35 Convent Drive, MSC 3703, Bethesda, MD 20892-3703. E-mail: bellusc@@inds.nih.gov.

DOI:10.1523/JNEUROSCI.0053-11.2011

Copyright $\odot 2011$ the authors $\quad 0270-6474 / 11 / 3111905-09 \$ 15.00 / 0$
}

mouse olfactory system an excellent in vivo model to study the process of axon guidance and neuronal connectivity.

Emerging evidence shows that apoptotic mechanisms contribute to the regulation of neuronal growth cone dynamics (Gilman and Mattson, 2002; Nikolaev et al., 2009). In the visual system, caspase- 3 activation has been reported to regulate chemotropic responses of growth cones in vitro (Campbell and Holt, 2003). In the olfactory system, signaling from apoptotic protease activating factor 1 (Apaf1) and caspase- 9 affects the embryonic development of OSNs and their axons (Ohsawa et al., 2010). These findings suggest that regulators of the apoptotic pathways may have the potential to modulate neuronal axon guidance.

A common apoptotic signaling pathway used during development is initiated by the death receptor Fas (Choi and Benveniste, 2004), but its involvement in axon guidance is unclear. Fas-associated factor 1 (FAF1) plays a role as an apoptotic signaling regulator since it interacts with Fas and can enhance Fas-mediated apoptosis in cells (Chu et al., 1995; Betarbet et al., 2008). Interestingly, FAF1 is expressed in the developing nervous system including the olfactory system during late embryonic development, correlating with the period of glomerular map formation (De Zio et al., 2008). These observations make FAF1 a compelling candidate molecule for studying the function of apoptotic signaling in axon guidance within the olfactory system.

To determine the role of FAF1 in axon guidance, we have generated transgenic mice in which FAF1 is selectively expressed in a subpopulation of OSNs as they seek to form connections with their targets in the OB. Here we report that FAF1 overexpression not only alters global OSN axonal projections with axons overextending to the deep layers of the $\mathrm{OB}$, but also disrupts specific OSN glomerular convergence with axons rerouted to incorrect 
glomerular targets. These findings clearly show a novel function for FAF1 in axon guidance.

\section{Materials and Methods}

Mice. The full-length coding region of the mouse FAF1 gene was subcloned into a standard TetO expression vector with the internal ribosome entry site (IRES) sequence and the green fluorescent protein (GFP) reporter gene (Gogos et al., 2000). TetO-FAF1 (TetO-FAF1IRES-GFP) transgenic lines were generated by pronuclear injection of zygotes from $\mathrm{FVB} / \mathrm{N}$ mice as previously described (Nguyen et al., 2007). Primers 5'-TGACCTCCATAGAAGAC ACC- ${ }^{\prime}$ and $5^{\prime}$-AAAGTCACTTTGTAGAAT GC-3' were used to genotype the mutant allele. The PCR was conducted with 35 cycles of reaction $\left(94^{\circ} \mathrm{C}\right.$ for $30 \mathrm{~s}, 55^{\circ} \mathrm{C}$ for $30 \mathrm{~s}, 72^{\circ} \mathrm{C}$ for $\left.30 \mathrm{~s}\right)$ using MangoTaq DNA polymerase (Bioline USA). G G $_{\gamma}$-tTA mice were a generous gift from Dr. Nicholas Ryba (The National Institute of Dental and Craniofacial Research). P2-IREStaulacZ (P2-ETL) mice were purchased from The Jackson Laboratory. Mice of both sexes were used for these experiments.

In situ hybridization. The standard procedure was performed as described previously (Schaeren-Wiemers and Gerfin-Moser, 1993). Briefly, fresh tissues of postnatal day 10 (P10) mouse heads were frozen in O.C.T. compoundembedding medium, and $16 \mu \mathrm{m}$ coronal sections were prepared using a Cryostat (Leica), collected on slides, and fixed in $4 \%$ paraformaldehyde for $20 \mathrm{~min}$. In situ hybridization was performed using a digoxigeninlabeled RNA probe corresponding to mouse FAF1 cDNA C-terminal region (nucleotides $1831-1950$ ). The slides were hybridized at $65^{\circ} \mathrm{C}$ overnight. The signal was detected with alkaline phosphatase-conjugated anti-digoxigenin antibody (Roche) at $4^{\circ} \mathrm{C}$ and with NBT/BCIP (Promega) solutions at room temperature.

Immunohistochemistry. Heads of mouse embryos were fixed in $4 \%$ paraformaldehyde, cryoprotected, and frozen in O.C.T. compoundembedding medium before $20 \mu \mathrm{m}$ parasagittal sections were cut using a cryostat and were collected on slides. Sections were subjected to antigen retrieval by immerging slides into citrate buffer, $\mathrm{pH}$ 6, heated to $95^{\circ} \mathrm{C}$ for $10 \mathrm{~min}$. After antigen retrieval, immunohistochemistry was performed on slides with FAF1 antibody (1:200, Protein Tech Group) using DAB (Vector Laboratories). Three-week-old and 12-week-old mice were perfused with $4 \%$ paraformaldehyde. Tissues of the $\mathrm{OE}$ and the $\mathrm{OB}$ were dissected and embedded in $10 \%$ gelatin. The embedded tissues were postfixed, cryoprotected, and frozen before the $40 \mu \mathrm{m}$ coronal sections were prepared using a cryostat. Immunohistochemistry of the floating sections was performed as previously described (Marks et al., 2006). Primary antibodies used were anti-growth-associated protein 43 (GAP43) (1:1000, Novus Biologicals), anti-olfactory marker protein (OMP) (1:5000, Wako Chemicals), anti-FAF1 (1:1000, Protein Tech Group), anti-cleaved caspase-3 (1:1000, Cell Signaling Technology), anti- $\beta$-galactosidase ( $\beta$-gal) (1:1000, ICN Biomedicals). Cy3-conjugated secondary antibodies were used at a 1:1000 dilution (Jackson ImmunoResearch). Confocal images ( $1 \mu \mathrm{m}$ optical sections) were collected using a Zeiss LSM-510-Meta confocal microscope. Fluorophores used were GFP (excitation 488, emission 507) and Cy3 (excitation 550, emission 570). Ectopic glomeruli were determined as the coalescence of $\mathrm{OMP}+$ axons found isolated in the external plexiform layer (EPL) beneath the GL, with an obvious gap between them and the GL. The boundary between the GL and EPL was defined using the basal edge of periglomerular cells shown by DAPI staining.

Western blot analysis. The OEs of 3-week-old mice were dissected and homogenized in lysis buffer ( $50 \mathrm{~mm}$ Tris, $\mathrm{pH} 7.5,150 \mathrm{~mm} \mathrm{NaCl}, 0.25 \%$ sodium deoxycholate, 1 mm EDTA, $0.5 \%$ Nonidet P-40) with protease inhibitor cocktail (Roche). The protein extracts were separated by 4-20\% gradient SDS-PAGE (Lonza), transferred to nitrocellulose membrane (PerkinElmer). Afterward, Western blots were detected by FAF1 antibody (1:1000, Santa Cruz Biotechnology).

Real-time PCR. The OEs of 2-week-old mutant mice $(n=4)$ and their littermate controls $(n=4)$ were collected and fresh frozen. The tissues were pooled together, and the total RNA from mutants and controls were extracted using Trizol reagent (Life Technologies) followed by phenol and chloroform extraction. The first strand cDNA was generated using SuperScript III (Life Technologies). Real-time PCR was performed using TaqMan Gene Expression Assays and StepOne Real-Time PCR System (Life Technologies). The 18S rRNA was used as the endogenous control. Littermate controls were used as the reference samples. The relative expression levels of faf1 and phosphoglycerate kinase 1 (pgk1) mRNA in mutants compared with controls were measured by real-time PCR.

Whole-mount lacZ staining. Mouse heads at different ages were dissected and cut through the midline to expose the medial surface of the OE and the OB. Tissues were subsequently fixed in $4 \%$ paraformaldehyde for 30 min on ice. Heads were washed twice with buffer A (PBS containing 2 $\mathrm{mM} \mathrm{MgCl}_{2}$ ) for 15 min each time, and once with buffer B (PBS containing $2 \mathrm{mM} \mathrm{MgCl}_{2}, 0.01 \%$ sodium deoxycholate and $0.02 \%$ Nonidet P-40) for $5 \mathrm{~min}$. Mouse heads were placed in the lacZ staining solution [buffer B with $5 \mathrm{~mm}$ potassium hexacyanoferrate (II) trihydrate, $5 \mathrm{~mm}$ potassium ferricyanide, and $0.2 \mathrm{mg} / \mathrm{ml} 5$-bromo-4-chloro-3-indolyl- $\beta$-Dgalactopyranoside] and shaken overnight at $32^{\circ} \mathrm{C}$. The reaction was stopped by $4 \%$ paraformaldehyde. Images were taken using a SPOT digital camera (Diagnostic Instruments) mounted to a dissection microscope (Leica).

Recovery study. When a new litter was born, the nursing mother was immediately placed on doxycycline (DOX) diet ( $6 \mathrm{~g} / \mathrm{kg}$ DOX, Bio-Serv) for 3 weeks. DOX was delivered to the mouse pups through the milk. The OEs and the OBs were collected from the pups at 3 weeks for subsequent histological analysis.

Cell counts. For P2 OSN cell counts, after whole-mount lacZ staining, the pictures of the medial view of the epithelium were taken using a SPOT digital camera mounted to a dissection microscope as described in whole mount lacZ staining. Labeled P2 OSNs from turbinates 2 and 3 were 

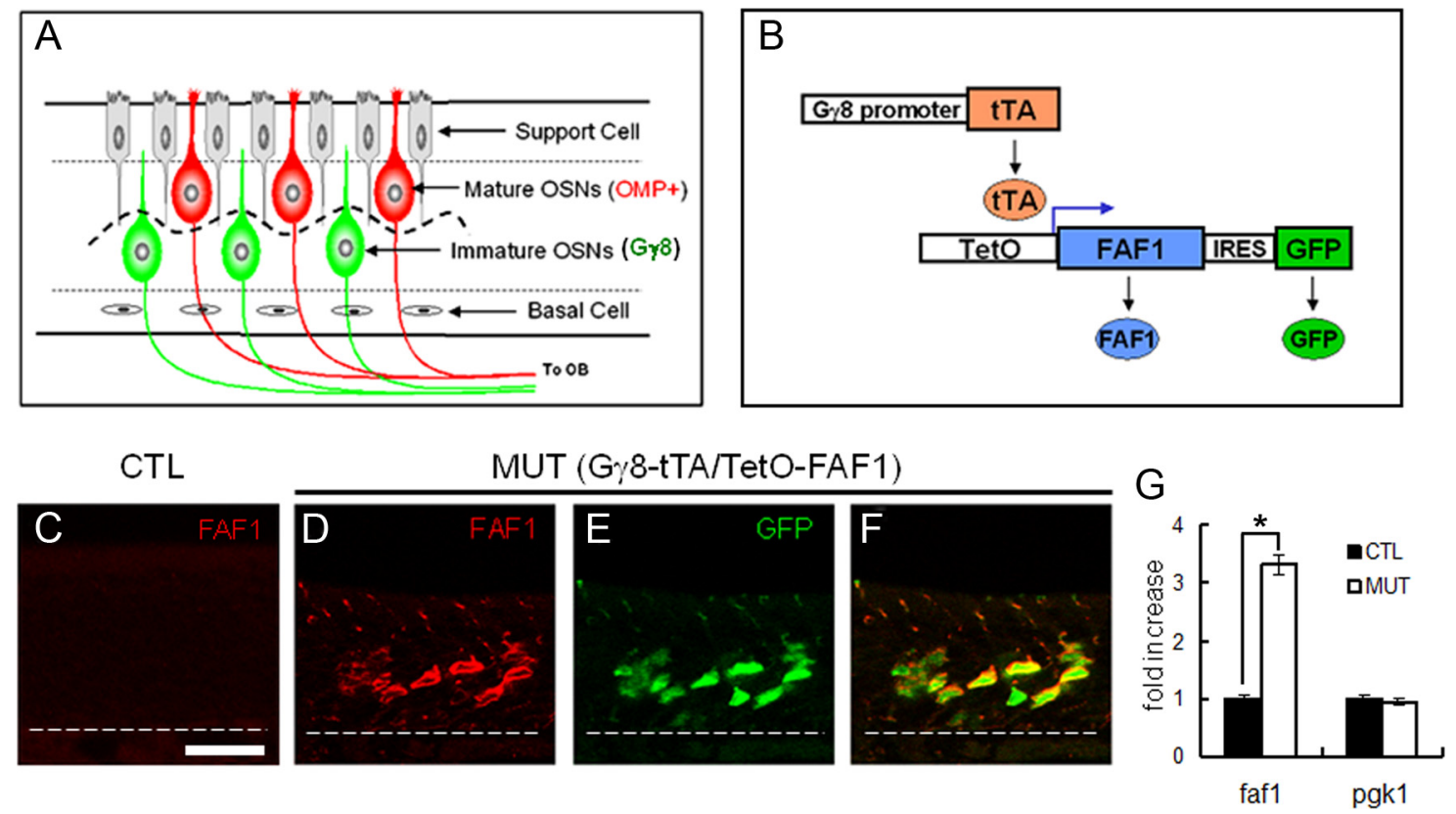

Figure 2. FAF1 can be overexpressed in immature 0SNs using the tTA system. $A$, Schematic of different cell types in the mouse 0 E. $B$, Schematic of exogenous FAF1 expression in mouse 0SNs using the tTA system by crossing $G_{\gamma} 8$-tTA and Tet0-FAF1. C, Very low levels of endogenous FAF1 expression in 12-week-old control (Tet0-FAF1) 0E is observed by immunohistochemistry. D-F, FAF1 protein $(\boldsymbol{D}$, red) and GFP $(\boldsymbol{E}$, green) are coexpressed $(\boldsymbol{F}$, overlap) in immature 0SNs of the 12-week-old mutant (G $\gamma$-tTA/Tet0-FAF1) 0E. $\boldsymbol{G}$, The relative expression level of faf1 transcript from 2-week-old control and mutant mouse epithelium is measured by real-time PCR. There is a significant increase $\left({ }^{*} p<0.001 ; t=-105.527\right)$ of faf1 transcript expression in the 2-week-old mutant mouse epithelium $(3.32 \pm 0.05 ; n=4)$ compared with controls $(1 \pm 0.02 ; n=4)$. pgk1 is used as a control, and its transcript expression is not significantly changed $(p=0.013 ; t=3.464)$ in mutants $(0.94 \pm 0.03 ; n=4)$ compared with controls $(1 \pm 0.03 ; n=4)$. CTL, Control; MUT, mutant. Scale bar, $50 \mu \mathrm{m}$. The dashed lines in $\boldsymbol{C}-\boldsymbol{F}$ separate the $0 \mathrm{E}$ and the lamina propria. Error bars represent \pm SD.

counted manually and pooled for each individual. For active caspase-3 cell counts for each animal, three representative pictures of cleaved caspase-3 antibody-immunostained coronal sections sampled from the anterior, middle, and posterior regions of the $\mathrm{OE}$ along the septum were taken using a digital camera (Olympus America) mounted to a fluorescence microscope (Carl Zeiss). The number of cleaved caspase-3immonoreactive cells per $5 \mathrm{~mm}$ length of epithelium along the septum was counted manually and averaged among three representative pictures for each individual. OMP + and GAP43 + cell counts: After coronal sections of the OE were immunostained with OMP antibody and GAP43 antibody, three representative single optical section $(1 \mu \mathrm{m})$ confocal images sampled from anterior, middle, and posterior regions of the $\mathrm{OE}$ along the septum were taken using a confocal microscope (Carl Zeiss). The numbers of OMP + and GAP43 + cells per $0.1 \mathrm{~mm}$ length of the OE along the septum were counted using Volocity Image Analysis Software (PerkinElmer) and were averaged among three representative images for each individual. Statistical analysis was performed using the $t$ test for the comparison between two groups and using the one-way ANOVA followed by the Holm-Sidak post hoc test for the comparison among the three groups.

\section{Results}

\section{FAF1 expression during olfactory development}

We detected clear FAF1 protein expression at embryonic day 14.5 (E14.5) in both the OE and the OB (Fig. 1A). These results concur with transgenic experiments suggesting that FAF1 is highly expressed in the mouse olfactory system at embryonic stages (De Zio et al., 2008) corresponding to the initial period of the glomerular map formation. Although we observed FAF1 protein expression throughout the epithelium, expression levels were not uniform with some cells exhibiting higher levels of FAF1 than others (Fig. $1 B$ ). Interestingly, FAF1 protein is present both in cell bodies and in the axon bundles of sensory neurons (Fig. $1 A, B$, arrows), suggesting a possible functional role of FAF1 in axon development. As postnatal FAF1 levels decrease with only low levels of RNA and protein expression maintained in the $\mathrm{OE}$ through adulthood (Figs. 1C,D, 2C) (De Zio et al., 2008), the transcript expression also becomes ubiquitously distributed in all OSNs (Fig. 1C). These data show that FAF1 is indeed expressed in OSN axons and its expression levels are high during early development when OSN axons are targeting regions of the OB. Thus, we hypothesized that altering FAF1 expression might lead to changes in the wiring of OSN axons within the OB.

\section{Genetic engineering of mice overexpressing FAF1 in immature OSNs}

In the mouse OE, OSNs can be divided into two broad categories, mature and immature, based in part upon their cell body location and marker protein expression (Fig. 2A). Mature OSNs are located in the apical layers of the OE and express the OMP (Farbman and Margolis, 1980; Miragall and Monti Graziadei, 1982). Most axons of these mature OSNs have either started to form or have formed synapses with secondary neurons within glomeruli (Farbman and Margolis, 1980). By comparison, immature OSNs are typically located in the basal portion of the OE and express proteins such as GAP43 and a G-protein subunit, $\mathrm{G}_{\gamma} 8$ (Verhaagen et al., 1989; Ryba and Tirindelli, 1995; Tirindelli and Ryba, 1996). Axons from these immature OSNs are actively searching for their targets and are typically found in the olfactory nerve layer of the $\mathrm{OB}$ with some entering the glomerular layer. This immature stage of OSN development is critical for axon guidance and is necessary to ensure proper glomerular targeting.

The tetracycline-inducible system is an ideal tool for selectively turning on/off a transgene expression at specific time points (Zhu et al., 2002). We used this system in our study by generating a series of transgenic mouse lines $(n=2)$ in which the FAF1 coding region is placed under the control of the synthetic promoter TetO and a bicistronic GFP reporter is introduced 
downstream under the control of an IRES cassette (TetO-FAF1-IRES-GFP or TetOFAF1). These lines were then crossed with a $\mathrm{G}_{\gamma} 8$-tTA transactivator line (previously described by Nguyen et al., 2007) to generate $\mathrm{G}_{\gamma} 8$-tTA/TetO-FAF1 mutants (Fig. $2 B)$. We analyzed these two compound transgenic lines and observed high levels of FAF1 protein in OSNs located at the basal part of the epithelium (Fig. 2D), compared with control or wild-type cells (Fig. 2C). In the mutant OEs, FAF1 immunostaining colocalized well with the GFP reporter (Fig. 2D-F), and the GFP colocalized with the immature OSN marker GAP43 (Fig. 3D). In addition, we also observed a large increase in FAF1 transcript expression in the mutant $\mathrm{OE}$ compared with controls using real-time PCR (Fig. 2G). Although we failed to observe FAF1 expression in mature OSNs through either immunohistochemistry or GFP expression, we cannot rule out the possibility that low levels of FAF1 may be present in mature neurons. Nevertheless, we conclude that by using the tetracycline-inducible system, we can accurately and effectively overexpress FAF1 in immature OSNs.

\section{Altered olfactory epithelium in mutant mice overexpressing FAF1}

Histological analysis of the OEs from 3-week-old $\mathrm{G}_{\gamma} 8$-tTA/TetO-FAF1 mice revealed an immediate phenotype. The thickness of the OE was clearly reduced in mutant mice compared with control mice

(Fig. 3A-F). To determine which population of OSNs was affected by FAF1 expression, we performed immunohistochemical staining with a series of marker proteins, including GAP43 to label immature OSNs (Fig. $3 A, D$ ) and OMP to mark mature OSNs (Fig. $3 B, E$ ). We quantified the numbers of GAP43- and OMP-immunoreactive cells per $0.1 \mathrm{~mm}$ length of $\mathrm{OE}$ along the septum. The numbers of GAP43-positive neurons between mutant mice $(39.6 \pm 4.6$; mean $\pm \mathrm{SD} ; n=12)$ and controls $(42.4 \pm$ 4.9; mean $\pm \mathrm{SD} ; n=9$ ) showed no significant difference $(p=$ $0.195 ; t=1.344$ ) (Fig. $3 G$ ). However, the number of OMPpositive OSNs significantly decreased $\left(\sim 46 \%\right.$ less, ${ }^{\star} p<0.001$; $t=10.894)$ in mutant mice $(35.9 \pm 3.1$; mean $\pm \mathrm{SD} ; n=5)$ compared with controls $(66.0 \pm 5.5$; mean $\pm \mathrm{SD} ; n=6)$ (Fig. $3 H)$. The loss of mature OSNs suggested that FAF1 might produce increased cell death within the mutant OE. We therefore examined the levels of apoptosis in the OE using active (cleaved) caspase-3 immunostaining (Fig. 3C,F) and counted the number of immunoreactive cells per $5 \mathrm{~mm}$ length of epithelium along the septum. As expected, the number of dying cells significantly increased $\left(\sim 82 \%\right.$ more; $\left.{ }^{\star} p<0.001 ; t=-4.565\right)$ in the OEs of mutant mice $(43.8 \pm 9.1$; mean $\pm \mathrm{SD} ; n=6)$ compared with controls $(24.1 \pm 8.4 ;$ mean $\pm \mathrm{SD} ; n=12)$ (Fig. 3I). Interestingly, we found no dying cells that clearly overlapped with the GFP expressing immature neurons (Fig. $3 F$ ), suggesting that the OSNs are lost after FAF1 expression is turned off. These data demon-
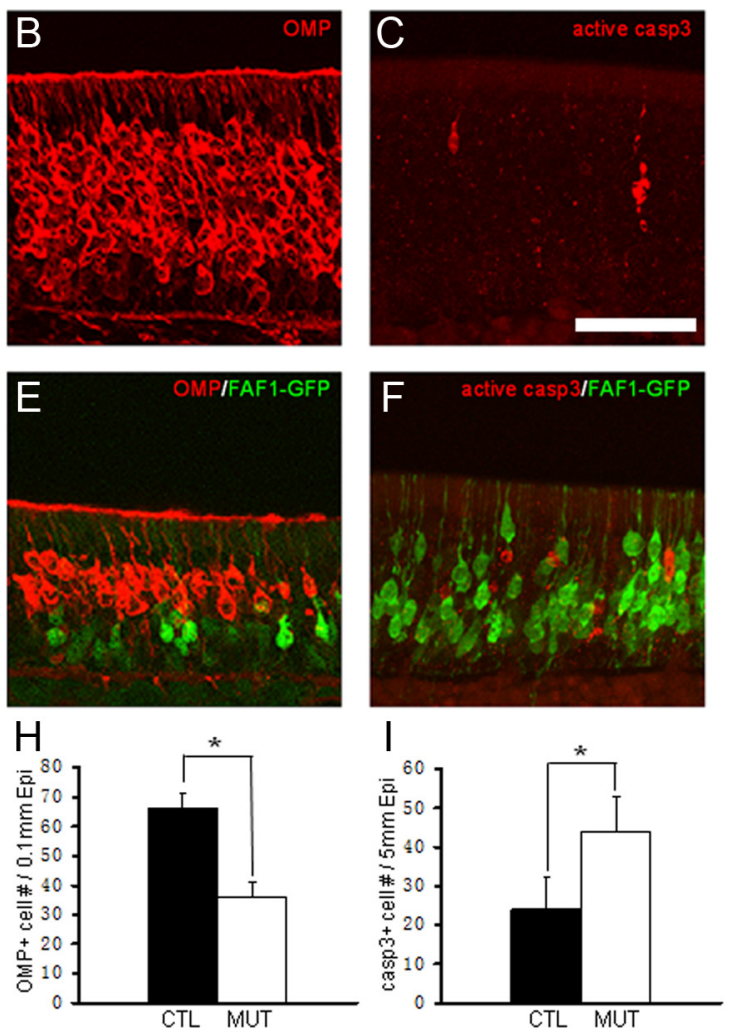

Figure 3. FAF1 overexpression causes loss of mature OSNs but does not directly induce apoptosis in immature 0SNs. $\boldsymbol{A}-\boldsymbol{F}$, Immunohistochemistry is performed in mouse $0 \mathrm{E}$ at P21. The expression of GAP43 $(\boldsymbol{A}, \boldsymbol{D}), 0 \mathrm{OMP}(\boldsymbol{B}, \boldsymbol{E})$, and active caspase-3 $(\boldsymbol{C}, \boldsymbol{F})$

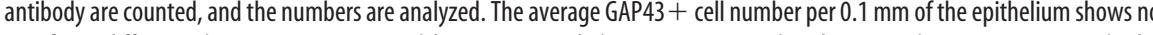
average $0 \mathrm{MP}+$ cell number per $0.1 \mathrm{~mm}$ of epithelium decreases significantly $\left({ }^{*} p<0.001 ; t=10.894\right)$ in mutants $(35.9 \pm 3.1$; $n=5)$ compared with controls $(66.0 \pm 5.5 ; n=6)$. The average active caspase- $3+$ cell number per $5 \mathrm{~mm}$ of epithelium increases significantly $\left({ }^{*} p<0.001 ; t=-4.565\right)$ in mutants $(43.8 \pm 9.1 ; n=6)$ compared with controls $(24.1 \pm 8.4 ; n=12)$. n.s., Not significant; casp3, caspase-3; Epi, epithelium; CTL, control; MUT, mutant. Scale bar, $50 \mu \mathrm{m}$. Error bars represent \pm SD.

strate that FAF1 overexpression in immature OSNs does not directly cause cell death.

\section{FAF1 overexpression causes distortion of olfactory bulb circuitry}

While expression of FAF1 protein is readily detectable in immature OSN cell bodies, it was unclear whether overexpressed FAF1 protein can distribute to immature axon terminals as they seek to find their glomerular targets. Using immunohistochemical staining in 3-week-old $\mathrm{G}_{\gamma} 8$-tTA/TetO-FAF1 mice, we found that FAF1 protein is broadly distributed within the olfactory nerve layer (Fig. $4 E, F$ ) and detectable in some axon terminals entering glomeruli (Fig. $4 E$, arrowheads). Thus, we sought to determine whether this axonal expression had any consequence on glomerular formation or layer organization within the OB. To assess this, we evaluated the general anatomical structure of the glomerular layer using OMP immunohistochemistry in mature OSN axons. As OMP immunostaining highlights most mature glomeruli in the main $\mathrm{OB}$, it is a reliable indicator of glomerular structure. We immediately identified several striking developmental defects associated with the OBs of FAF1-expressing mutants. First, we observed entire regions that were devoid of glomeruli in the mutant bulbs (Fig. 4N,O). Second, we found that in mutant mice many OSN axons project to the wrong layers of the OB. In controls, OMP-positive axons travel through the olfactory nerve layer at the edge of the $\mathrm{OB}$ and terminate neatly within the glo- 

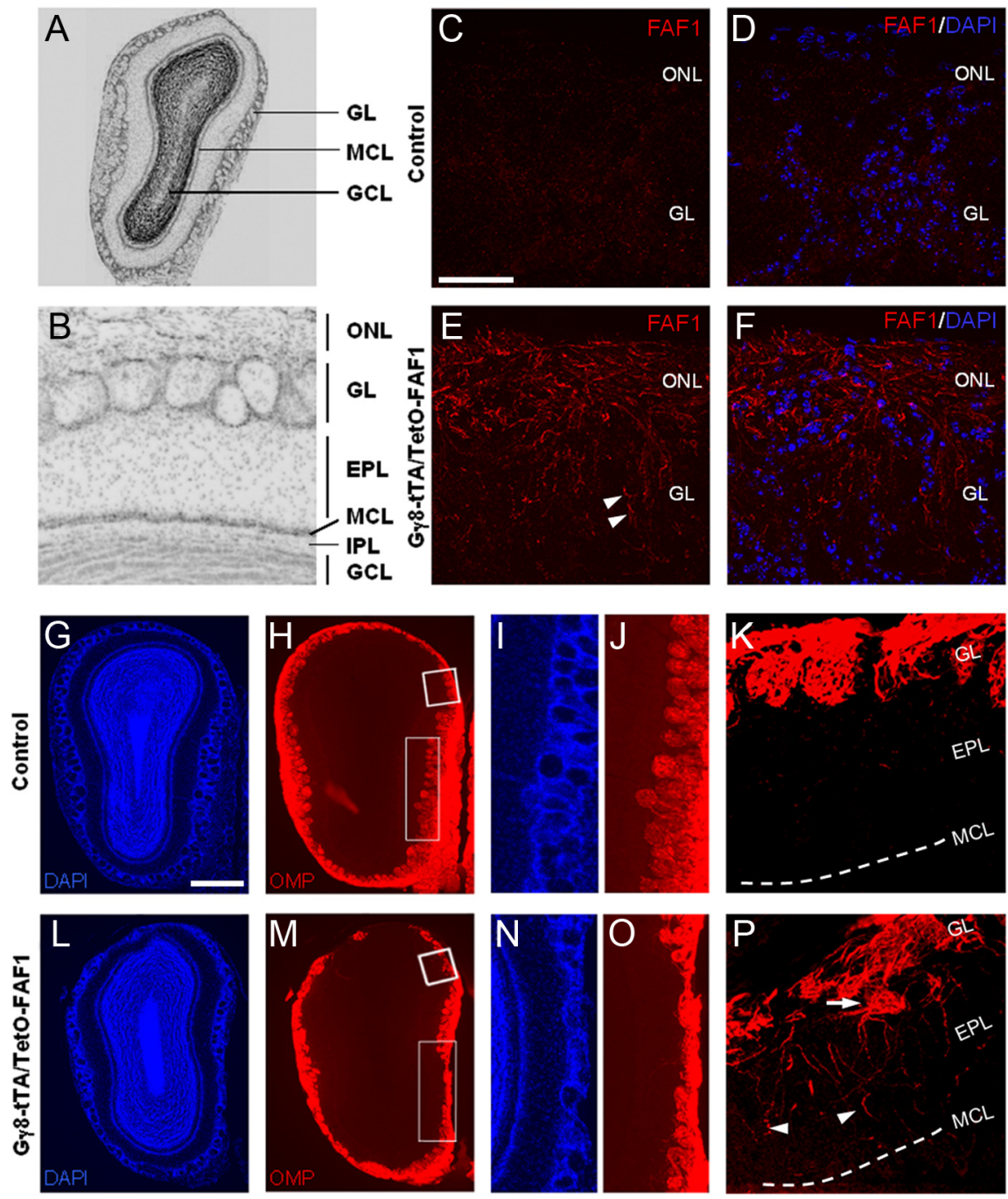

Figure 4. FAF1 overexpression causes glomerular structure malformation and OSN axon overshooting. $A, B$, Schematic of a coronal section of the $\mathrm{OB}$ indicating the layers. ONL, Olfactory nerve layer; IPL, internal plexiform layer; $\mathrm{GCL}$, granule cell layer. $\boldsymbol{C}-\boldsymbol{F}$, Overexpressed FAF1 protein can be detected by immunohistochemistry at the OSN axon terminal in the ONL $(\boldsymbol{E})$ and GL $(\boldsymbol{E}$, arrowheads) on $0 B$ coronal sections of the mutant $\mathrm{G}_{\gamma} 8$-tTA/Tet0-FAF1 $(\boldsymbol{E}, \boldsymbol{F})$ but not the control Tet0-FAF1 (C, D) at P21. G-P, The coronal sections of $\mathrm{OB}$ s are stained with $\operatorname{DAPI}(\boldsymbol{G}, \boldsymbol{I}, \boldsymbol{L}, \boldsymbol{N})$ and $\mathrm{OMP}(\boldsymbol{H}, \boldsymbol{J}, \boldsymbol{K}, \boldsymbol{M}, \mathbf{O}, \boldsymbol{P}) . \mathbf{G}-\boldsymbol{K}$, The control Tet0-FAF1 OB shows normal glomerular structure $(\boldsymbol{I}, \boldsymbol{J}$, close-up of rectangle boxed area in $\boldsymbol{H} ; \boldsymbol{K}$, close-up of square boxed area in $\boldsymbol{H})$. $\boldsymbol{L}-\boldsymbol{P}$, The mutant $\mathbf{G}_{\gamma} 8$-tTA/Tet0-FAF1 OB shows missing glomeruli $(\boldsymbol{N}, \mathbf{0}$, close-up of rectangle boxed area in $\boldsymbol{M})$, ectopic glomeruli (arrow in $\boldsymbol{P}$, close-up for square boxed area in $\boldsymbol{M}$ ), and overshooting 0 SN axons into the deep layers (arrowheads in $\boldsymbol{P}$ ). Scale bars: $(-\boldsymbol{F}, 50 \mu \mathrm{m}$; $\boldsymbol{G}, \boldsymbol{H}, \boldsymbol{L}, \boldsymbol{M}, 500 \mu \mathrm{m}$. The dashed lines in $\boldsymbol{K}$ and $\boldsymbol{P}$ indicate the position of the MCL.

merular layer, where they form synapses with the dendrites of mitral and tufted cells (Fig. $4 \mathrm{~K}$ ). However, in FAF1 mutant mice, the nerve layer is much thinner than in controls (Fig. $4 M, H$ ), and many OMP-positive axons overshoot into the deep layers of the OB such as the EPL and the mitral cell layer (MCL) (Fig. 4P, arrowheads). Third, in conjunction with the overshooting fibers, we also observed the formation of ectopic glomeruli in the EPL (Fig. $4 P$, arrow). These phenotypes clearly demonstrate that the general axon organization of OSNs is broadly altered by FAF1 overexpression.

\section{FAF1 overexpression disrupts axonal convergence of P2} OSNs

To study the effect of FAF1 overexpression on the targeting of specific OSN axons, we used the P2-ETL mouse line in which OSNs expressing the $\mathrm{P} 2$ odorant receptor are labeled with a $\beta$-galactosidase indicator fused with a tau protein (tauLacZ)
(Mombaerts et al., 1996). As a result, the P2 OSNs can be visualized both in their cell bodies and axons. By crossing $\mathrm{G}_{\gamma} 8$ tTA/TetO-FAF1 with P2-ETL mice, we could examine the effect of FAF1 overexpression on $\mathrm{P} 2$ OSNs. We first observed a sharp loss of P2 OSNs in the OEs of FAF1 mutants compared with controls at $\mathrm{P} 0$ (Fig. $5 B, E$ ), which remained significantly reduced at 3 weeks of age $\left({ }^{*} p<0.001 ; t=\right.$ 6.788 ) (Figs. $5 H, K, 6 M$ ). This was consistent with the decrease in OMP-positive OSNs in mutants (Fig. $3 \mathrm{H}$ ).

As previous studies showed that a reduction in P2 OSN number does not necessarily produce a disruption in axonal convergence (Lin et al., 2000; Cummings and Belluscio, 2010), we next examined the OBs. Interestingly, we immediately found extensive mistargeting of $\mathrm{P} 2$ axonal projections in FAF1 mutant mice. At $\mathrm{P} 0$, only a few wandering P2 neuron axons were detected in mutant OBs (Fig. $5 F$, arrows), compared with controls where glomerular convergence was clearly already established (Fig. 5C). At 3 weeks of age, the mutant phenotype progressed with P2 axons coalescing to form a string of glomerular-like structures across the surface of the bulb (Fig. $5 L$, arrows) rather than the stereotypic $\mathrm{P} 2$ glomerular convergence present in controls (Fig. 5I). Notably, this distorted P2 axonal pattern also persists into adulthood (data not shown). To better assess the degree of P2 OSN axonal mistargeting, we also stained coronal sections of OBs with $\beta$-gal antibody. This staining confirmed a characteristic set of one lateral (Fig. $5 \mathrm{M}, N$ ) and one medial P2 glomerulus (Fig. 5O,P) in controls. In FAF1 mutants, we identified a series of small glomerular-like structures on both lateral (Fig. 5Q,R) and medial (Fig. 5S,T) sides of the OB. Together, these findings show that FAF1 overexpression in immature OSNs produces a reduction in the number of $\mathrm{P} 2 \mathrm{OSN}$ and a disruption in their axonal convergence.

\section{DOX treatment reverses the effect of FAF1 on OSNs}

Since OSNs constantly regenerate, we next sought to determine whether the FAF1-induced broad disruption of OSN axonal projections is permanent or whether the endogenous organization can be recovered. Our mice were engineered with a tetracyclineinducible system that enables us to turn off FAF1 expression when the drug DOX is applied. Given that olfactory phenotypes were already evident at birth, we decided to shut down transgenic FAF1 expression immediately after birth. This was accomplished by feeding the nursing mother of the mutant mice a DOX diet that was transmitted to the mutant pups through their mother's milk. Animals were maintained on the DOX diet from P0 to P21, at which point we confirmed that FAF1 overexpression was efficiently turned off (Fig. 6A-D). We then performed this recovery 
paradigm on FAF1 mutant mice containing the P2-ETL allele to assess the effect on axonal targeting. Strikingly, we found that at $\mathrm{P} 21$ the P2 OSN axons reconverge to form a major $\mathrm{P} 2$ glomerulus in the $\mathrm{OB}$ shown by whole-mount lacZ staining (Fig. 6E,F). Immunohistochemical data further confirmed that a primary P2 glomerulus is re-established on the lateral (Fig. 6G, arrow) and medial (Fig. 6H, arrow) sides of the $\mathrm{OB}$, with smaller minor glomeruli only occasionally detectable (Fig. 6G, arrowhead). At a broader anatomical level, we observed that the characteristic OMP-positive overshooting axons were also completely eliminated and that the glomerular structure was restored (Fig. 6I). In the OE, we found that the numbers of P2 neurons (Fig. 6J), OMPpositive OSNs (Fig. $6 K$ ), and active caspase-3-positive cells (Fig. 6L) all returned to control levels (Figs. $3 \mathrm{~B}, \mathrm{C}, 5 \mathrm{H}$ ) (Fig. $6 \mathrm{M}$ shows cell numbers as a percentage of controls). Together, these data clearly illustrate that the broad range of phenotypes exhibited by the OE and the $\mathrm{OB}$ caused by FAF1 overexpression are all reversible following reduction of FAF1 levels.

\section{Discussion}

FAF1 as a regulator of axon guidance Formation of the olfactory map requires the cooperative integration of many guidance molecules and signaling pathways (Sakano, 2010). Here we report that a molecule that is unlike other identified factors in olfactory axon guidance, the apoptosis regulator FAF1, can also contribute to OSN axon pathfinding. Although apoptosis is generally thought of as a molecular mechanism used to eliminate cells, there is increasing evidence supporting nonlethal roles for some apoptotic molecules (Kuranaga and Miura, 2007). In the nervous system, nonapoptotic activity of caspase- 3 has been reported to regulate dendritic spine degeneration in hippocampal neurons (D'Amelio et al., 2011). Interestingly, components of the apoptotic machinery, from the upstream receptors (e.g., death receptor 6 and p75NTR neurotrophic receptor) to the downstream effectors (e.g., caspase-6 and caspase-3), have all been shown to be present in nerve terminals that are focal points for axon guidance (Gilman and Mattson, 2002; Campbell and Holt, 2003; McLaughlin, 2004; Carson et al., 2005; Nikolaev et al., 2009). Studies have demonstrated that the apoptotic pathway can locally mediate chemotropic responses of retinal growth cones (Campbell and Holt, 2003), raising the interesting possibility that apoptotic molecules may play a role in regulating the axon guidance process. Furthermore, a recent study also showed that Apaf1 and $J, 1 \mathrm{~mm} ; M, \mathbf{0}, \mathbf{Q}, \mathbf{S}, 500 \mu \mathrm{m}$.
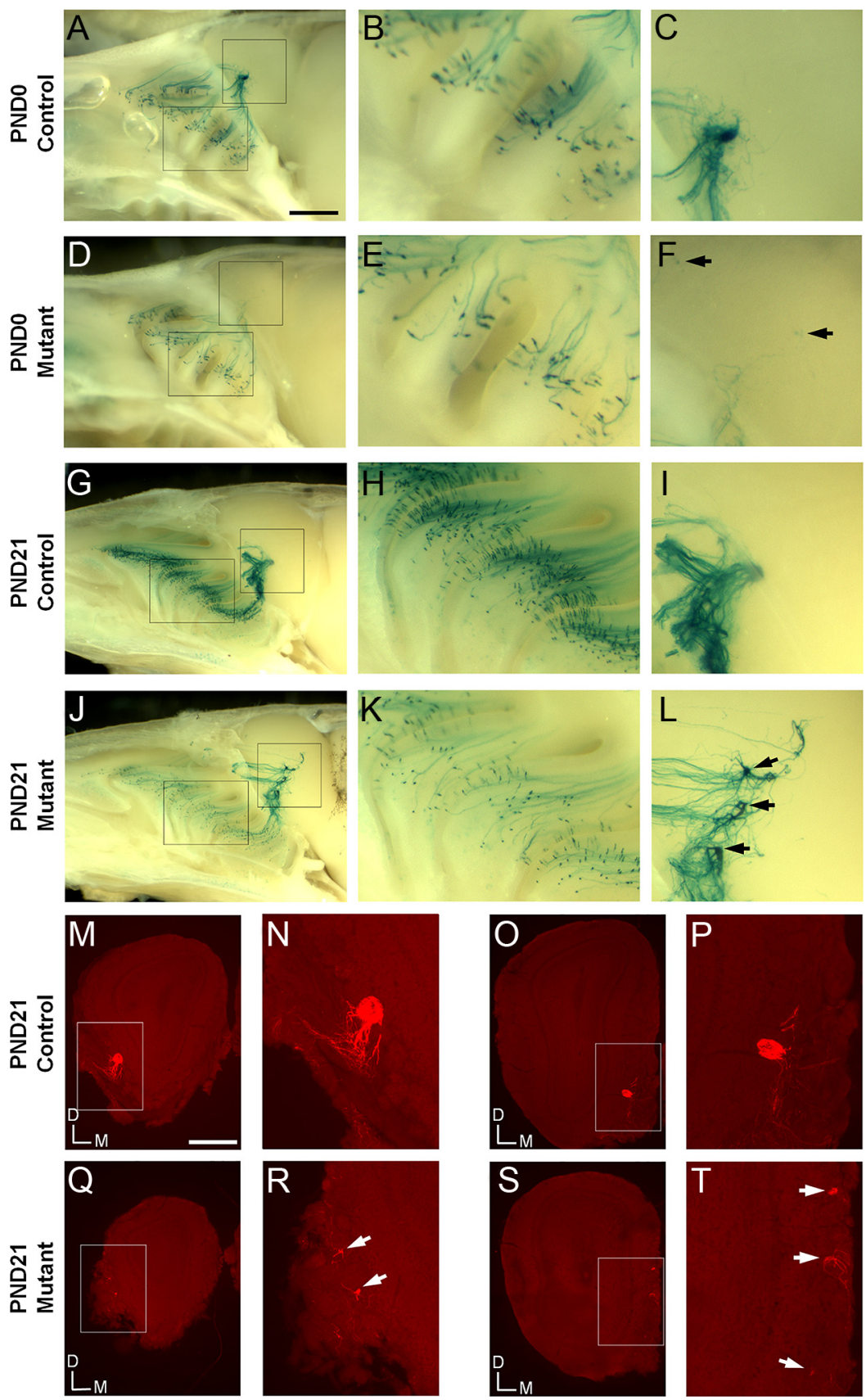

Figure 5. FAF1 overexpression disrupts P2 OSN axonal convergence. All controls are Tet0-FAF1/P2-ETL ${ }^{+/-}$, and all mutants are $\mathrm{G}_{\gamma} 8$-tTA/Tet0-FAF1/P2-ETL ${ }^{+/-}$. $A-L$, The whole-mount lacZ staining of mouse $0 \mathrm{E}$ and $0 \mathrm{~B}$ reveal P2-lacZ OSN axonal projections in controls $(\boldsymbol{A}-\boldsymbol{C}, \boldsymbol{G}-\boldsymbol{I})$ and mutants $(\boldsymbol{D}-\boldsymbol{F}, \boldsymbol{J}-\boldsymbol{L})$ at $\mathrm{PO}(\boldsymbol{A}-\boldsymbol{F})$ and $\mathrm{P} 21(\boldsymbol{G}-\boldsymbol{L})$, respectively. The close-ups of the $0 \mathrm{E}$ (rectangular area) and the $O B$ (square area) in the left panels $(\boldsymbol{A}, \boldsymbol{D}, \boldsymbol{G}, \boldsymbol{J})$ are shown in the middle panels $(\boldsymbol{B}, \boldsymbol{E}, \boldsymbol{H}, \boldsymbol{K})$ and right panels $(\boldsymbol{C}, \boldsymbol{F}, \boldsymbol{I}, \boldsymbol{L})$, respectively. While controls show well formed $P 2$ glomeruliat $P 0(C)$ and $P 21(I)$, mutants show wandering $P 2$ axons on the surface of the $\mathrm{OB}$ at $\mathrm{PO}$ ( $\boldsymbol{F}$, arrows) and a string of small glomerular-like structures at P21 ( $\boldsymbol{L}$, arrows). The P2 neuron number decreases in mutants $(\boldsymbol{E}, \boldsymbol{K})$ compared with controls $(\boldsymbol{B}, \boldsymbol{H}) \cdot \boldsymbol{M}-\boldsymbol{T}, \boldsymbol{\beta}$-gal immunostaining is performed on $0 \mathrm{~B}$ coronal sections of control $(\boldsymbol{M}-\boldsymbol{P})$ and mutant $(\mathbf{Q}-\boldsymbol{T})$ mice at $\mathbf{P 2 1}$. The close-ups of the boxed region $(\boldsymbol{M}, \mathbf{O}, \mathbf{Q}, \mathbf{S})$ are shown on its right $(\boldsymbol{N}, \boldsymbol{P}, \boldsymbol{R}, \boldsymbol{T})$, respectively. The immunohistochemistry further confirms the widespread small $P 2$ glomerular-like structures on both lateral ( $\boldsymbol{R}$, arrows) and medial $(\boldsymbol{T}$, arrows) sides of the mutant $\mathrm{OBs}$, compared with typical P2 glomeruli in controls ( $N, P)$. D, Dorsal; $M$, medial. Scale bars: $A, D, G$,

caspase-9 knock-out mouse embryos exhibit impaired maturation of OSNs and altered projections of P2 OSN axons (Ohsawa et al., 2010). While the OSN targeting phenotypes in this study were small and limited to prenatal stages due to perinatal lethality (Kuida et al., 1998; Yoshida et al., 1998), they demonstrated that apoptotic signaling is playing a role for proper formation of OSN axonal connec- 


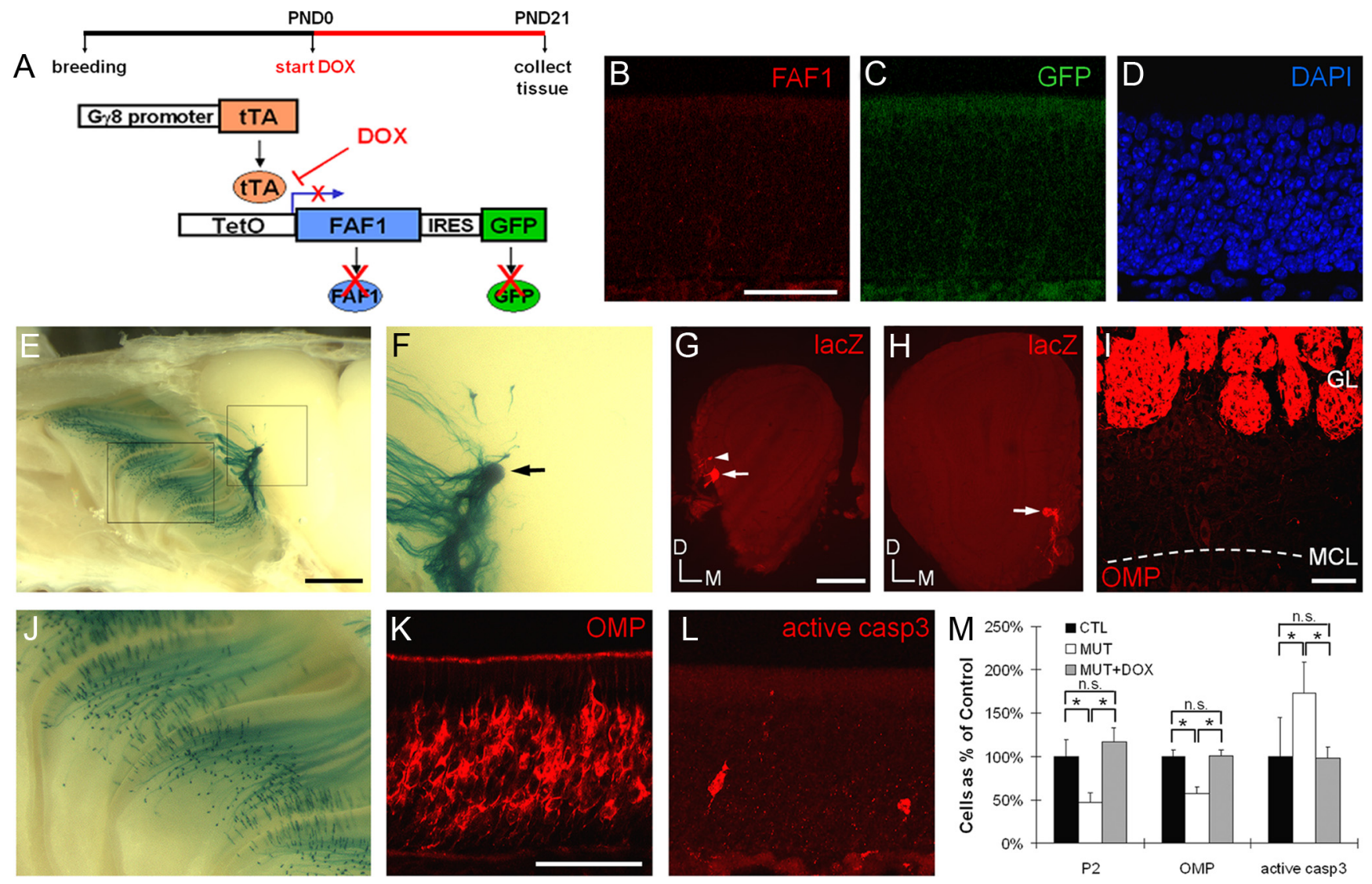

Figure 6. Disrupted olfactory bulb and epithelium in $\mathrm{G}_{\gamma} 8$-tTA/Tet0-FAF1 mice can be restored by DOX treatment. $A$, Schematic for turning off exogenous FAF1 expression with DOX treatment. All OE and OB images are from P21 $\mathrm{G}_{\gamma} 8$-tTA/Tet0-FAF1/P2-ETL ${ }^{+/-}$mutant mice treated with a DOX diet from P0 to P21. $\boldsymbol{B}-\boldsymbol{D}$, show complete shutdown of transgene using FAF1 antibody $(\boldsymbol{B})$ and GFP reporter $(\boldsymbol{C})$ at $\mathrm{P} 21$ with DAPI showing nuclear staining $(\boldsymbol{D})$. $\boldsymbol{E}-\boldsymbol{I}$, Recovery of the $\mathrm{OB}$. Whole-mount lacZ staining shows restoration of $\mathrm{P} 2$ glomeruli $(\boldsymbol{E}, \boldsymbol{F}$, close-up of the $0 \mathrm{~B}$, arrow). $\beta$-gal immunostaining of $O B$ coronal sections also confirms this restoration of the lateral $(\boldsymbol{G}$, arrow) and medial ( $\boldsymbol{H}$, arrow) P2 glomeruli, with occasional minor P2 glomeruli detectable ( $\boldsymbol{G}$, arrowhead). $\boldsymbol{I}$, $O M P$ immunostaining of $O B$ coronal sections reveals the recovery of layers with the elimination of overshooting axons deep into the $O B$. $J-M$, Recovery of the $0 E$. Whole mount lacZ staining of the $O E$ shows recovery in number of $P 20 S N s$ ( $\boldsymbol{J}$, close-up of the $0 E$ in $E$ ). Immunostaining of 0 E sections reveals restoration of OMP + neurons $(\boldsymbol{K})$, and a decrease in active caspase-3-expressing cells $(\boldsymbol{L})$. M, Quantitative comparison of P2 OSNs, OMP-positive cells, and active caspase-3-positive cells in control, mutant, and D0X-treated mutant mice at P21. The graph shows the cell number as a percentage of controls (100\%). The number of P2-ETL OSNs in FAF1 mutant mice $(88.9 \pm 20.1$; graphed as $47.6 \pm 10.8 \% ; n=8)$ is significantly lower than both controls $(186.8 \pm 36.8$; graphed as $\left.100 \% \pm 19.7 \% ; n=10 ;{ }^{*} p<0.001 ; t=6.788\right)$ and age-matched mutants treated with $\operatorname{DOX}\left(219.0 \pm 29.7 ;\right.$ graphed as $\left.117.2 \pm 15.9 \% ; n=5 ;{ }^{*} p<0.001 ; t=7.506\right)$. However, there is no significant difference in P2-ETL OSN numbers between DOX-treated mutants and controls ( $p=0.0675, t=1.933$ ). Similarly, the average number of OMP + cells per $0.1 \mathrm{~mm}$ of epithelium in mutants $(35.9 \pm 3.1$; graphed as $54.4 \pm 4.7 \% ; n=5)$ significantly decreases compared with both controls $\left(66.0 \pm 5.5 ;\right.$ graphed as $\left.100 \% \pm 8.3 \% ; n=6 ;{ }^{*} p<0.001 ; t=10.904\right)$ and DOX-treated mutants $\left(67.0 \pm 4.5\right.$; graphed as $\left.101.5 \pm 6.8 \% ; n=6 ;{ }^{*} p<0.001 ; t=11.273\right)$. However, the numbers of OMP + cells is not significantly different between D0X-treated mutants and controls ( $p=0.705 ; t=0.387$ ). Conversely, the average number of active caspase- $3+$ cells per $5 \mathrm{~mm}$ of epithelium in mutants $(43.8 \pm 9.1 ;$ graphed as $181.7 \pm 37.8 \% ; n=6)$ increases significantly compared with both controls $\left(24.1 \pm 8.4 ;\right.$ graphed as $\left.100 \% \pm 34.9 \% ; n=12 ;{ }^{*} p<0.001 ; t=5.668\right)$ and D0X-treated mutants $(24.8 \pm 3.2 ;$ graphed as $102.9 \pm 13.3 \% ; n=12$; $\left.{ }^{*} p<0.001 ; t=5.452\right)$. However, the numbers of active caspase-3 + cells in DOX-treated mutants is not significantly different from controls ( $\left.p=0.794 ; t=0.264\right)$. n.s., Not significant; D, dorsal; M, medial; Casp3, caspase-3; CTL, Tet0-FAF1/P2-ETL ${ }^{+/-}$control; MUT, G ${ }_{\gamma} 8$-tTA/Tet0-FAF1/P2-ETL ${ }^{+/-}$mutant; MUT+DOX, G ${ }_{\gamma}^{8-t T A / T e t 0-F A F 1 / P 2-E T L ~}{ }^{+/-}$mutant treated with a D0X diet from P0 to P21. Scale bars: $\boldsymbol{B}-\boldsymbol{D}, \boldsymbol{I}, \boldsymbol{K}, \boldsymbol{L}, 50 \mu \mathrm{m} ; \boldsymbol{E}, 1 \mathrm{~mm} ; \boldsymbol{G}, \boldsymbol{H}, 500 \mu \mathrm{m}$. The dashed line in $\boldsymbol{I}$ indicates the position of MCL. Error bars represent \pm SD.

tions. In our study, we provide unique in vivo evidence that the apoptosis regulator, FAF1, can indeed modulate the axon guidance process during postnatal development.

Initially identified as Fas-associated factor, very little is known about this molecule and its role in apoptosis (Chu et al., 1995). While it has been indicated in the formation of the deathinducing signaling complex (Ryu et al., 2003), the exact mechanism by which the proposed Fas-FAF1 signaling can lead to cell death is still unclear. Previous findings have shown that FAF1 expression in the developing nervous system is dynamic, including prominent expression in the embryonic olfactory system (De Zio et al., 2008) (Fig. 1 $A, B$ ), which later falls during postnatal stages (De Zio et al., 2008) (Fig. 2C; data not shown). This expression pattern is consistent with a role for FAF1 in axon guidance, as many OSN axons must find targets in the $\mathrm{OB}$ during early development while postnatally this demand decreases with the maturation of the glomerular map. To investigate the potential role of FAF1 in axon targeting, we maintain high levels of FAF1 expression in OSNs through birth and into the postnatal period, resulting in broad disruption of glomerular structures (Fig. 4) with overextension of mature OSN axons deep into the OB (Fig. $4 P$ ) and the specific distortion of P2 OSN axonal convergence (Fig. 5). Together, these data all support an axon guidance function for FAF1.

\section{FAF1 overexpression results in loss of mature OSNs}

Recent studies support the notion that FAF1 may not be a "killer" of cells, but rather a regulator of apoptosis (Ryu et al., 2003; Kinoshita et al., 2006; Betarbet et al., 2008). In the OE of FAF1 mutants, we observed cell loss in mature OSNs (Fig. $3 B, E, H$ ) but not in immature OSNs with high levels of FAF1 (Fig. $3 A, D, G$ ). This suggests that FAF1 expression does not directly cause cell 
death but rather plays another role, possibly regulating axon guidance that may eventually predispose cells to apoptosis due to secondary factors (Fig. 3), although we cannot completely rule out the possibility that OSNs may initiate their cell death program while still immature. As OSNs develop, they extend axons to target specific glomeruli in the OB. Upon reaching their glomerular targets, developing OSNs turn off immature markers (e.g., G $\gamma 8$ and GAP43), and turn on mature markers (e.g., OMP) as they form synaptic connections with mitral and tufted cells (Farbman and Margolis, 1980; Verhaagen et al., 1989; Tirindelli and Ryba, 1996). Interestingly, this brief immature-to-mature transition period when OSNs are first establishing postsynaptic contacts and thus are only beginning to express OMP is a critical stage when OSNs are very susceptible to apoptosis (Carson et al., 2005). Thus, it is possible that the mature cell loss identified in FAF1 mutants is simply a consequence of immature axonal mistargeting. Although, we note that any residual FAF1 protein persisting after the shutdown of $\mathrm{G} \gamma 8$ expression may also contribute to the apoptotic process.

\section{FAF1-induced disorganization is reversible}

Mouse glomerular map formation is a complex process involving both dorsal-ventral and anterior-posterior patterning. Studies have shown that dorsal-ventral patterning is partially mediated by molecules such as Robo2/Slit1 and Nrp2/Sema3F expressed as gradients, while anterior-posterior patterning is regulated by odorant receptor-derived cAMP signals determining the expression of Nrp1/Sema3A and PlxnA1/Sema6C gradients as well as segregation molecules like Kirrel2/Kirrel3, BIG2, and EphA/ephrinA (Cho et al., 2009; de Castro, 2009; Imai et al., 2010; Sakano, 2010). Despite these extensive studies on glomerular map formation, it is still unclear whether the glomerular map can be restored following broad axonal disruption.

To address the issue of map restoration, studies have used chemical agents or surgical procedures to lesion the olfactory nerve and have shown that while OSNs do regenerate and extend axons to the $\mathrm{OB}$, the glomerular map remains distorted (Costanzo, 2000; John and Key, 2003). This may be due to a basic inability of OSN axons to correctly restore the glomerular map or may be a consequence of physical damage associated with the experimental approach, such as scarring. By contrast, one study used a genetic approach to selectively eliminate only a subpopulation of OSNs that express the P2 odorant receptor and found that upon regeneration the $\mathrm{P} 2$ glomerulus returned, intact (Gogos et al., 2000). One explanation for this discrepancy is that since the genetic ablation affected only the P2 OSNs, the residual axons from these ablated OSNs left a molecular trail, an "invisible track" for the regenerating neurons to follow upon their axonal targeting. If so, then broad disruption of axonal projections through a genetic approach should scramble these tracks and thus bias regenerating axons toward a distorted glomerular organization. Using our FAF1 mutant mice, we tested this hypothesis and found that the P2 glomeruli can be accurately restored even after broad glomerular disruption (Figs. 4-6), demonstrating that the paths of previous OSN axons do not determine the organization of the glomerular map.

Another explanation for our observed map recovery is that the mechanisms necessary for glomerular map formation persist beyond initial OB development and, as long as significant tissue disruption does not occur (e.g., chemical or surgical ablation), the regenerating axons can use endogenous cues to accurately restore order to the map. In this scenario, reorganization would occur in conjunction with OSN turnover: as old mistargeted
OSNs die, new OSNs replace them with correctly targeted axons. Such a model is consistent with our data, demonstrating that the disrupted P2 glomeruli can regenerate in 3 weeks during the highly proliferative early postnatal period (Fig. $6 F$ ). Although small ectopic $\mathrm{P} 2$ glomeruli occasionally persist and likely require a longer recovery period to be fully eliminated (Fig. 6G, arrowhead). In the OE, we note that the number of P2 OSNs also returns to control levels upon FAF1 reduction, along with the number of OMP-positive and active caspase-3-prositive cells (Fig. 6J-M). Similarly, in the OB we find that glomerular structure and layer organization also return to normal (Fig. $6 F-I$ ), all consistent with the ability of olfactory projections to accurately restore themselves.

\section{FAF1 as a multifunctional protein}

We have shown a novel function of FAF1 in axon guidance beyond its traditional role in cell death. While there is little doubt that FAF1 can indeed regulate apoptotic signaling, it is worth noting that FAF1 can also modulate a variety of other cellular processes including nuclear factor $-\kappa \mathrm{B}$ signaling (Park et al., 2004) and the ubiquitination pathway (Ryu and Kim, 2001; Song et al., 2005). Perhaps the most striking evidence that FAF1 plays an important developmental role is that the FAF1 knock-out mice are embryonic lethal at the two cell stage, revealing that its role is fundamental (Adham et al., 2008). Intriguingly, FAF1 expression has also been associated with neurological disorders such as Alzheimer's disease $(\mathrm{AD})$ and Parkinson's disease $(\mathrm{PD})$ with the FAF1 gene locus linked to late-onset PD (Hicks et al., 2002). Studies have found elevated levels of FAF1 protein in brain tissues from both $\mathrm{AD}$ and PD patients with FAF1 expression directly colocalizing with neurons positive for $\alpha$-synuclein, a critical component in PD pathology (Betarbet et al., 2008). Similarly, in vitro experiments show that PD-related insults increase FAF1 expression in cultured cells (Betarbet et al., 2008), suggesting that FAF1 may also be a player of the neurodegenerative process.

In summary, FAF1 appears to be a multifunctional protein that modulates various cellular processes in addition to apoptotic signaling. Our study demonstrates that FAF1 functions as a regulator of axon guidance possibly in conjunction with neurodevelopment. While determining the precise function of FAF1 in each of these processes is challenging, perhaps through the utilization of FAF1 conditional knockouts it may reveal the underlying mechanisms.

\section{Notes}

Supplemental material for this article is available at http://data.ninds.nih. gov/. In vitro cultures of dissociated OSNs. This material has not been peer reviewed.

\section{References}

Adham IM, Khulan J, Held T, Schmidt B, Meyer BI, Meinhardt A, Engel W (2008) Fas-associated factor (FAF1) is required for the early cleavagestages of mouse embryo. Mol Hum Reprod 14:207-213.

Axel R (1995) The molecular logic of smell. Sci Am 273:154-159.

Betarbet R, Anderson LR, Gearing M, Hodges TR, Fritz JJ, Lah JJ, Levey AI (2008) Fas-associated factor 1 and Parkinson's disease. Neurobiol Dis 31:309-315.

Campbell DS, Holt CE (2003) Apoptotic pathway and MAPKs differentially regulate chemotropic responses of retinal growth cones. Neuron 37:939-952.

Carson C, Saleh M, Fung FW, Nicholson DW, Roskams AJ (2005) Axonal dynactin p150Glued transports caspase-8 to drive retrograde olfactory receptor neuron apoptosis. J Neurosci 25:6092-6104.

Cho JH, Prince JE, Cloutier JF (2009) Axon guidance events in the wiring of the mammalian olfactory system. Mol Neurobiol 39:1-9. 
Choi C, Benveniste EN (2004) Fas ligand/Fas system in the brain: regulator of immune and apoptotic responses. Brain Res Brain Res Rev 44:65-81.

Chu K, Niu X, Williams LT (1995) A Fas-associated protein factor, FAF1, potentiates Fas-mediated apoptosis. Proc Natl Acad Sci U S A 92:11894-11898.

Costanzo RM (2000) Rewiring the olfactory bulb: changes in odor maps following recovery from nerve transection. Chem Senses 25:199-205.

Cummings DM, Belluscio L (2010) Continuous neural plasticity in the olfactory intrabulbar circuitry. J Neurosci 30:9172-9180.

D’Amelio M, Cavallucci V, Middei S, Marchetti C, Pacioni S, Ferri A, Diamantini A, De Zio D, Carrara P, Battistini L, Moreno S, Bacci A, Ammassari-Teule M, Marie H, Cecconi F (2011) Caspase-3 triggers early synaptic dysfunction in a mouse model of Alzheimer's disease. Nat Neurosci 14:69-76.

de Castro F (2009) Wiring olfaction: the cellular and molecular mechanisms that guide the development of synaptic connections from the nose to the cortex. Front Neurosci 3:52.

De Zio D, Ferraro E, D’Amelio M, Simoni V, Bordi M, Soroldoni D, Berghella L, Meyer BI, Cecconi F (2008) Faf1 is expressed during neurodevelopment and is involved in Apaf1-dependent caspase-3 activation in proneural cells. Cell Mol Life Sci 65:1780-1790.

Farbman AI, Margolis FL (1980) Olfactory marker protein during ontogeny: immunohistochemical localization. Dev Biol 74:205-215.

Gilman CP, Mattson MP (2002) Do apoptotic mechanisms regulate synaptic plasticity and growth-cone motility? Neuromolecular Med 2:197-214.

Gogos JA, Osborne J, Nemes A, Mendelsohn M, Axel R (2000) Genetic ablation and restoration of the olfactory topographic map. Cell 103:609-620.

Hicks AA, Pétursson H, Jónsson T, Stefánsson H, Jóhannsdóttir HS, Sainz J, Frigge ML, Kong A, Gulcher JR, Stefánsson K, Sveinbjörnsdóttir S (2002) A susceptibility gene for late-onset idiopathic Parkinson's disease. Ann Neurol 52:549-555.

Imai T, Sakano H, Vosshall LB (2010) Topographic mapping-the olfactory system. Cold Spring Harb Perspect Biol 2:a001776.

John JA, Key B (2003) Axon mis-targeting in the olfactory bulb during regeneration of olfactory neuroepithelium. Chem Senses 28:773-779.

Kinoshita T, Kondoh C, Hasegawa M, Imamura R, Suda T (2006) Fasassociated factor 1 is a negative regulator of PYRIN-containing Apaf-1like protein 1. Int Immunol 18:1701-1706.

Kuida K, Haydar TF, Kuan CY, Gu Y, Taya C, Karasuyama H, Su MS, Rakic P, Flavell RA (1998) Reduced apoptosis and cytochrome c-mediated caspase activation in mice lacking caspase 9. Cell 94:325-337.

Kuranaga E, Miura M (2007) Nonapoptotic functions of caspases: caspases as regulatory molecules for immunity and cell-fate determination. Trends Cell Biol 17:135-144.

Lin DM, Wang F, Lowe G, Gold GH, Axel R, Ngai J, Brunet L (2000) Formation of precise connections in the olfactory bulb occurs in the absence of odorant-evoked neuronal activity. Neuron 26:69-80.

Marks CA, Cheng K, Cummings DM, Belluscio L (2006) Activity-dependent plasticity in the olfactory intrabulbar map. J Neurosci 26:11257-11266.

McLaughlin B (2004) The kinder side of killer proteases: caspase activation contributes to neuroprotection and CNS remodeling. Apoptosis 9:111-121.
Miragall F, Monti Graziadei GA (1982) Experimental studies on the olfactory marker protein. II. Appearance of the olfactory marker protein during differentiation of the olfactory sensory neurons of mouse: an immunohistochemical and autoradiographic study. Brain Res 239:245-250.

Mombaerts P, Wang F, Dulac C, Chao SK, Nemes A, Mendelsohn M, Edmondson J, Axel R (1996) Visualizing an olfactory sensory map. Cell $87: 675-686$.

Nguyen MQ, Zhou Z, Marks CA, Ryba NJ, Belluscio L (2007) Prominent roles for odorant receptor coding sequences in allelic exclusion. Cell 131:1009-1017.

Nikolaev A, McLaughlin T, O'Leary DD, Tessier-Lavigne M (2009) APP binds DR6 to trigger axon pruning and neuron death via distinct caspases. Nature 457:981-989.

Ohsawa S, Hamada S, Kuida K, Yoshida H, Igaki T, Miura M (2010) Maturation of the olfactory sensory neurons by Apaf-1/caspase-9-mediated caspase activity. Proc Natl Acad Sci U S A 107:13366-13371.

Park MY, Jang HD, Lee SY, Lee KJ, Kim E (2004) Fas-associated factor-1 inhibits nuclear factor-kappaB (NF-kappaB) activity by interfering with nuclear translocation of the RelA (p65) subunit of NF-kappaB. J Biol Chem 279:2544-2549.

Ryba NJ, Tirindelli R (1995) A novel GTP-binding protein gamma-subunit, G gamma 8 , is expressed during neurogenesis in the olfactory and vomeronasal neuroepithelia. J Biol Chem 270:6757-6767.

Ryu SW, Kim E (2001) Apoptosis induced by human Fas-associated factor 1, hFAF1, requires its ubiquitin homologous domain, but not the Fasbinding domain. Biochem Biophys Res Commun 286:1027-1032.

Ryu SW, Lee SJ, Park MY, Jun JI, Jung YK, Kim E (2003) Fas-associated factor 1, FAF1, is a member of Fas death-inducing signaling complex. J Biol Chem 278:24003-24010.

Sakano H (2010) Neural map formation in the mouse olfactory system. Neuron 67:530-542.

Schaeren-Wiemers N, Gerfin-Moser A (1993) A single protocol to detect transcripts of various types and expression levels in neural tissue and cultured cells: in situ hybridization using digoxigenin-labelled cRNA probes. Histochemistry 100:431-440.

Song EJ, Yim SH, Kim E, Kim NS, Lee KJ (2005) Human Fas-associated factor 1, interacting with ubiquitinated proteins and valosin-containing protein, is involved in the ubiquitin-proteasome pathway. Mol Cell Biol 25:2511-2524.

Tirindelli R, Ryba NJ (1996) The G-protein gamma-subunit G gamma 8 is expressed in the developing axons of olfactory and vomeronasal neurons. Eur J Neurosci 8:2388-2398.

Verhaagen J, Oestreicher AB, Gispen WH, Margolis FL (1989) The expression of the growth associated protein B50/GAP43 in the olfactory system of neonatal and adult rats. J Neurosci 9:683-691.

Yoshida H, Kong YY, Yoshida R, Elia AJ, Hakem A, Hakem R, Penninger JM, Mak TW (1998) Apaf1 is required for mitochondrial pathways of apoptosis and brain development. Cell 94:739-750.

Zhu Z, Zheng T, Lee CG, Homer RJ, Elias JA (2002) Tetracycline-controlled transcriptional regulation systems: advances and application in transgenic animal modeling. Semin Cell Dev Biol 13:121-128. 